\title{
Laparoscopic Resection of Retrocaval Non-functioning Paraganglioma
}

\author{
Moon Soo Lee, M.D. ${ }^{1}$, Yoon Jung Kang, M.D. ${ }^{1}$, Hyun-Young Han, M.D. ${ }^{2}$, \\ Hyun-Jin Son, M.D. ${ }^{3}$, Jae Min Lee, M.D. ${ }^{4}$ \\ Departments of ${ }^{1}$ Surgery, ${ }^{2}$ Radiology, ${ }^{3}$ Pathology, ${ }^{4}$ Internal Medicine, Eulji University Hospital, Daejeon, Korea
}

Retroperitoneal paraganglioma is located primarily at the para-aortic area and functioning tumor, however, non-functioning and retrocaval located tumors are rare. Laparoscopic retrocaval paraganglioma resection is technically challenging due to vena cava close relation. A 49-year-old male was referred for a retroperitoneal tumor, detected by FDG-PET scan. He had undergone right thyroidectomy for papillary carcinoma one year ago. Abdominal computed tomography (CT) scan showed a mass measuring $2 \mathrm{~cm}$ in size located behind the inferior vena cava (IVC), which deviate IVC anteriorly. $\mathrm{I}^{123}$-metaiodobenzylguanidine (MIBG) scan also demonstrated abnormal focal activity. Result of preoperative adrenal function test was normal. A laparoscopic retrocaval tumor excision was performed successfully. The operative time was 160 minutes. The patient's postoperative recovery was uneventful and he was discharged on the fourth postoperative day. Pathologic examination was consistent with the diagnosis of paraganglioma. To the best of our knowledge, this is the first report on laparoscopic resection of a retrocaval non-functioning paraganglioma. In conclusion, laparoscopic retrocaval paraganglioma resection is safe and feasible.

Key words: Paraganglioma, Non-functioning, Retrocaval, Laparoscopic

\section{INTRODUCTION}

Paragangliomas are extra-adrenal chromaffin tumors that develop at the expense of the neuroectodermal cells of the autonomous nervous system. Retroperitoneal and non-functioning forms are very rare. ${ }^{1}$ Laparoscopic adrenalectomy has been known as standard surgery for benign and partially malignant tumors. ${ }^{2}$ However, laparoscopic retrocaval paraganglioma resection is technically challenging due to its close relation to the vena cava. Accordingly, the laparotomy has been preferred. However, in our case, laparoscopic resection of the retrocaval paraganglioma was performed successfully. To our knowledge, this is the first report of the laparoscopic resection of a retrocaval non-functioning paraganglioma.

\section{CASE REPORT}

A 49-year-old male was referred for an asymptomatic retroperitoneal tumor, detected by FDG-PET. He underwent right thyroidectomy for a papillary carcinoma 1 year prior. During

Received December 19, 2013, Revised January 15, 2014,

Accepted February 24, 2014

※ Corresponding author : Moon Soo Lee

Departments of Surgery, Eulji University Hospital, 1306, Dunsan-

dong, Seo-gu, Daejeon 302-799, Korea

Tel : +82-42-259-1330, Fax : +82-42-259-1125

E-mail : mslee01@eulji.ac.kr the regular workup, FDG-PET revealed a mildly hypermetabolic, low density mass at the retrocaval area of the retroperitoneum (Fig. 1A). Abdominal computed tomography (CT) scan showed a $2 \mathrm{~cm}$-sized, round mass located directly behind the inferior vena cava (IVC), which deviated from the IVC anteriorly without definitive evidence of vessel-wall invasion (Fig. 1B). No perigastric or periesophageal lymph nodes were identified. An $\mathrm{I}^{123}$-metaiodobenzylguanidine (MIBG) scan also revealed abnormal focal activity in the right upper quadrant abdomen (Fig. 1C, D). The physical examination was unremarkable. The preoperative adrenal function test was within the normal range. A pre-operative evaluation led to the possible diagnosis of primary retroperitoneal tumor, such as a neurogenic tumor (more likely) or a thyroid cancer metastasis (less likely). A laparoscopic resection was scheduled for the non-functioning retrocaval tumor.

After the administration of general anesthesia, the patient was placed in a left lateral decubitus position at 90 degrees. A 12-mm trocar for the optic port was initially inserted at the anterior axillary line. After insufflation of the abdomen, two other 5-mm working ports were placed at the mid-axillary and mid-clavicular lines. Additionally, a 5-mm port was inserted at the epigastric area for liver retraction. All ports were placed at a two-finger breath distance apart along the right subcostal borders (Fig. 2). First, a triangular ligament of the liver and the adjacent IVC was dissected for mobilization of the liver. We then advanced to the retroperitoneal space through the right aspect of the IVC. The tumor was located behind the vena cava 

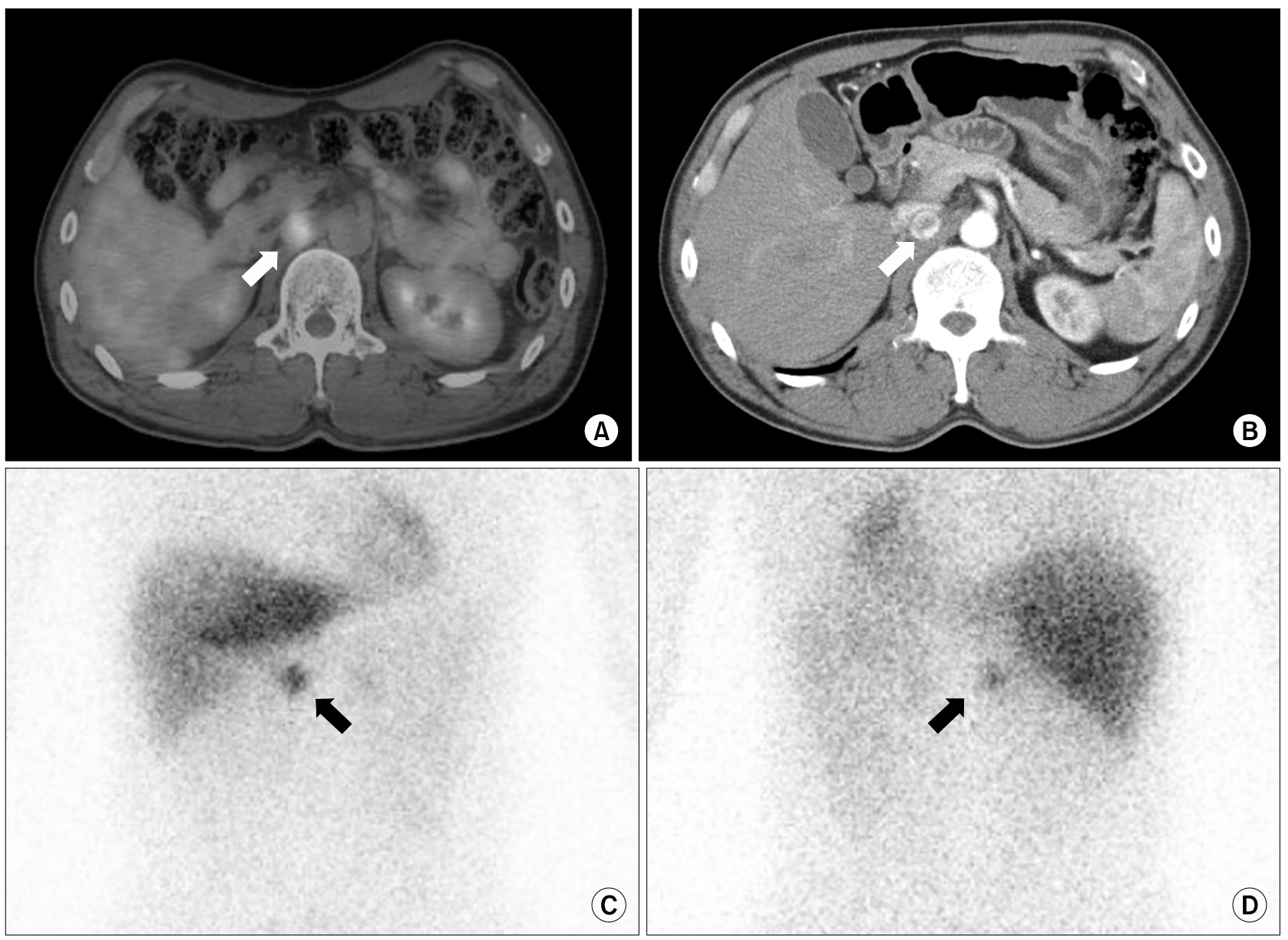

Fig. 1. (A) FDG-PET showing a mildly hypermetabolic, low density mass at retroperitoneum (retrocaval area). (B) Abdominal CT showing $2 \mathrm{~cm}$ round highly enhancing hypervascular mass with central necrosis in the retrocaval space, which deviated from IVC anteriorly without definitive evidence of vessel-wall invasion. (C, D) I- ${ }^{123}$ MIGB scan showing abnormal focal activity in right upper quadrant area.

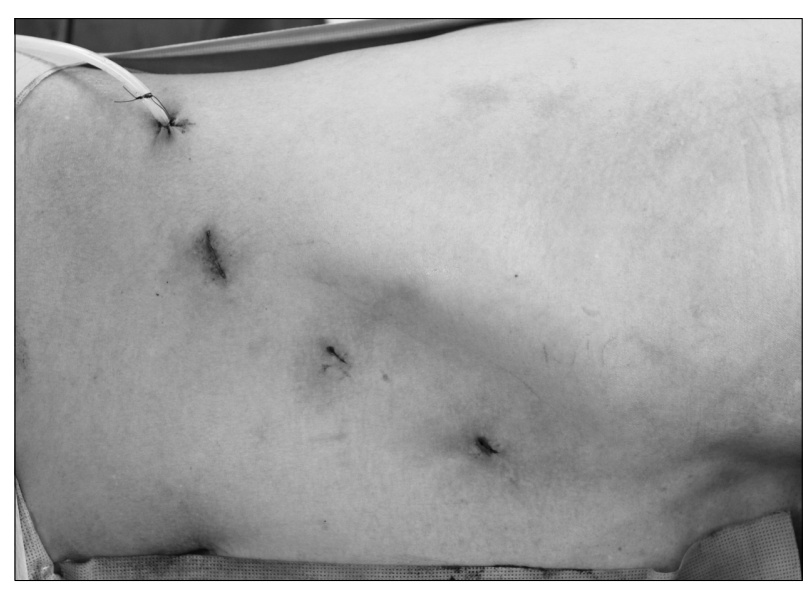

Fig. 2. Trocar site placements for the laparoscopic retrocaval tumor resection. and medial to lower pole of the adrenal gland The IVC was displaced and compressed anterio-medially by the tumor. After the localization of the tumor, the border between the anterior surface of the tumor and the posterior surface of the IVC was carefully dissected using a blunt dissector and a harmonic scalpel (Ethicon, Cincinnati, Ohio) (Fig. 3A). The tumor was progressively extracted from behind, on the right side of the vena cava. No direct invasion of the IVC was observed. Thereafter, we proceeded with dissection of the posterior attachment of the tumor. And the feeding vessel for the tumor was ligated and divided. Finally, the paraganglioma was divided from the paravertebral ganglia (Fig. 3B). The operative time was 160 minutes. During mobilization of the tumor, no elevations of the patient's blood pressure were observed. These procedures were successfully conducted without vascular injury and without conversion to open surgery. The patient's postoperative recov- 

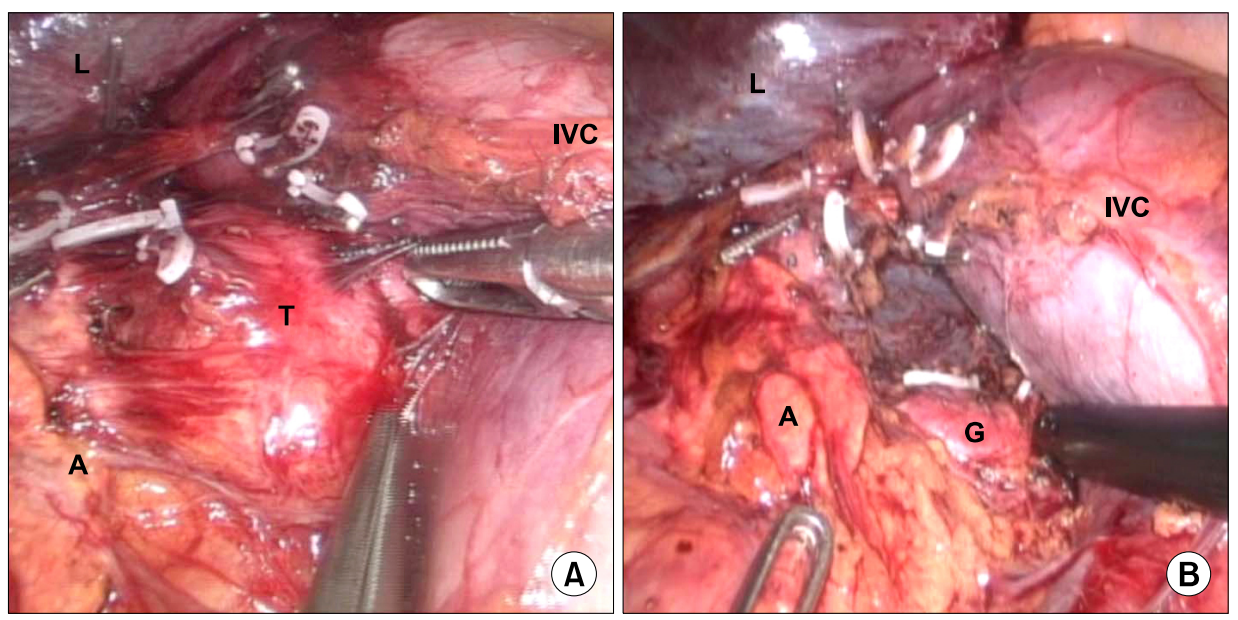

Fig. 3. (A) Laparoscopic view at the localization of retrocaval paraganglioma. (B) View after laparoscopic paraganglioma resection. $\mathrm{L}=$ liver; $\mathrm{A}=$ adrenal gland; $\mathrm{T}=$ tumor; $\mathrm{G}=$ paravertebral ganglia; $\mathrm{IVC}=$ inferior vena cava.

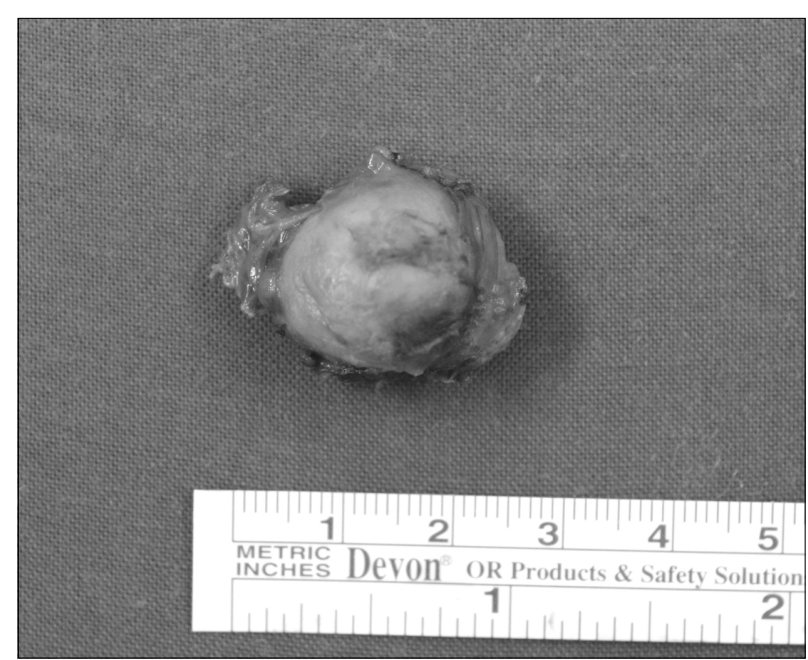

Fig. 4. Surgical specimen showed the grayish soft tissue, measuring $2.3 \times 2.0 \mathrm{~cm}$-sized mass.

ery was uneventful and he was discharged on the $4^{\text {th }}$ postoperative day. The surgical specimen showed $2.3 \times 2.0 \mathrm{~cm}$-sized, well-demarcated, soft mass (Fig. 4). Histological examination coupled with an immunohistochemical study was consistent with the diagnosis of paraganglioma with $\mathrm{Ki} 67<1 \%$ (Fig. 5). SDHB and SDHD exon mutation analyses were performed for exclusion of hereditary paraganglioma syndrome, because the patient suffered from papillary carcinoma and paraganglioma. DNA blood analysis revealed no SDHB and SDHD exon mutation.

\section{DISCUSSION}

According to the World Health Organization in 2004, paraganglioma is described as intra-adrenal paraganglioma, and is classified as pheochromocytoma and extra-adrenal paraganglioma. ${ }^{3}$ Paragangliomas is usually a functional tumor which secretes catecholamine or their metabolites. Functioning paraganglioma can be diagnosed from typical clinical symptoms such as palpitation and flushing face. However, a non-functional paraganglioma is very rare and the diagnosis may be a significant challenge because it is characterized by its asymptomatic profile and normal levels of catecholamines in the urine and blood. Therefore, a non-functional paraganglioma is usually discovered after the tumor has become sufficiently enlarged to produce symptoms related to the compression on adjacent organs. ${ }^{4}$ Also, non-functioning paraganglioma tumors tend to be difficult to resect due to their size and location. Computerized tomography has been established as the investigation method of choice for demonstrating the location, extent, and relation to surrounding tissues of a retroperitoneal tumor. Occasionally, as in our case, I- ${ }^{123}$ MIGB scan or PET-CT may be alternative diagnostic image modalities.

It may not be possible to differentiate benign from malignant paraganglioma tumors, even at the time of diagnosis. ${ }^{5}$ Therefore, complete surgical excision is considered as the treatment of choice. Laparoscopic adrenalectomy has become the standard approach for resection of benign adrenal diseases, including the pheochromocytoma. Also, open surgical procedures have been used to remove most extra-adrenal paragangliomas because they are mainly located at the para-aortic or para-caval area and are thus difficult to approach. ${ }^{6}$ However, recent improvements in minimally invasive surgery have made laparoscopic resection of paragangliomas possible. Tetsuo Nozaki et al. reported the first case of the laparoscopic resection of a retrocaval paraganglioma, demonstrating its feasibility, despite the high level of compression of the IVC. ${ }^{7}$ Also, Saud Alrasheedi 

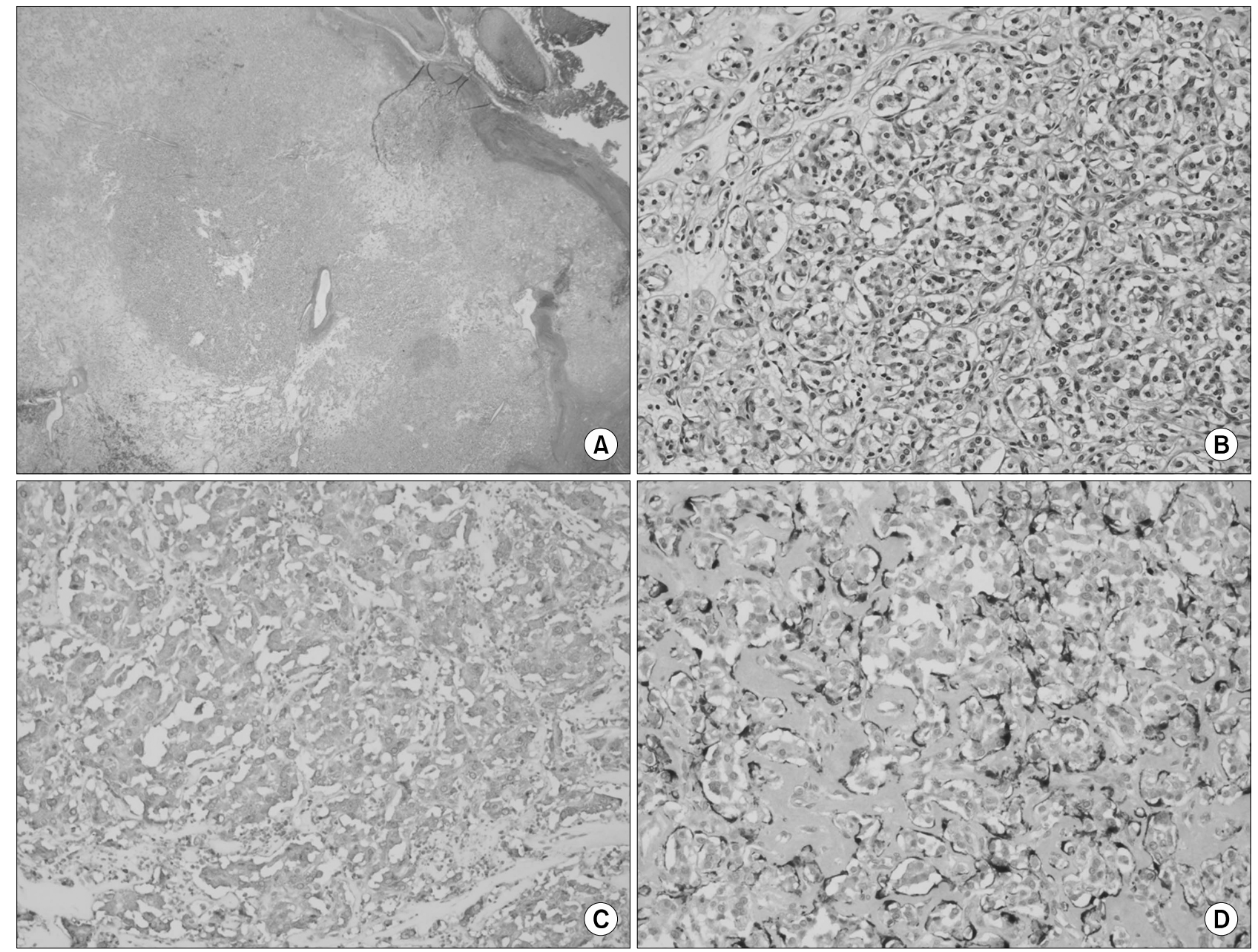

Fig. 5. Pathologic findings. (A) The tumor is well circumscribed and is separated by a fibrous capsule from the surrounding hypertrophic nerve bundles $(\mathrm{H} \& \mathrm{E}, \times 12.5)$. (B) Well-defined nests of cuboidal cells ("Zellballen") are separated by highly vascularized fibrous septa $(H \& E, \times 200)$. (C) Intense immunoreactivity for chromogranin in the cytoplasm of tumor cells (Chromogranin, $\times 200)$. (D) Sustentacular cells $($ S100 protein, $\times 200)$.

reported that a robotic approach as well as laparoscopic for retrocaval paraganglioma resection is feasible and safe. ${ }^{8}$ Most resection of laparoscopic or robotic retrocaval paraganglioma is confined to the functioning tumor. To our knowledge, this is the first case where a non-functioning paraganglioma located at the retrocaval area has been resected successfully laparoscopically.

The main risk of the laparoscopic approach for retrocaval tumor excision is injury and hemorrhage of the IVC during the tumor resection. The atraumatic grasper and $5 \mathrm{~mm}$ Endo peanut $^{\mathrm{TM}}$ (auto-suture) were useful for blunt dissection between the retrocaval tumor and the IVC. Most connective tissue and the feeding vessels were controlled using a harmonic scalpel and clips. In our case, no vessel invasion was observed due to the relatively small-size of the $(2 \mathrm{~cm})$ tumor. On the other hand, a more precise localization was needed for the small size tumor to avoid unnecessary dissection. This could be achievable using an anatomical landmark through the multimodality images at the preoperative preperation. If vessel-wall invasion is observed or the tumor is too large, laparoscopic resection of the paraganglioma should be considered inappropriate and the procedure should be converted to open surgery.

In conclusion, retroperitoneal retrocaval non-functioning paraganglioma is rare and may be difficult to diagnose. In addition, open surgical procedures were used to remove most extra-adrenal paraganglioma due to the difficulty in approaching the location. However, a laparoscopic resection of a retrocaval paraganglioma can be performed safely through proper patient selection and preoperative planning. 


\section{REFERENCES}

1) Hasbi S, El Khader A, El Fahssi $M$, et al. Case note: retroperitoneal nonfunctioning paraganglioma. Can J Surg 2010;53:3-4.

2) Smith CD, Weber CJ, Amerson JR. Laparoscopic adrenalectomy: new gold standard. World J Surg 1999;23:389-396.

3) DeLellis RA, Lloyd RV, Heitz PU, et al., eds. Pathology and Genetics of Tumours of Endocrine Organs. Lyon, France: IARC Press; 2004. World Health Organization Classification of Tumours.

4) Wen J, Li HZ, Ji ZG, et al. A case of large silent extra-adrenal retroperitoneal paraganglioma resected laparoscopically. Chin Med Sci J 2010;25:61-64.

5) John H, Ziegler W, Hauri D, et al. Pheochromocytomas: Can malignant potential be predicted? Urology 1999;53:679-683.

6) Tischler AS. Pheochromocytoma and extra-adrenal paraganglioma:Updates. Arch Pathol Lab Med 2008;132:1272-1284.

7) Nozaki $T$, Iida $H$, Tsuritani $S$, et al. Laparoscopic resection of retrocaval paraganglioma. J Laparoendosc Adv Surg Tech A 2010;20:363-367.

8) Alrasheedi S, Germain A, Zarngar R, et al. Robotic-assisted resection of a retrocaval paraganglioma. World Journal of Endocrine Surgery. Endocrine Image 2010;2:51-52. 